\title{
BMJ Open The influence of multisite pain and psychological comorbidity on prognosis of chronic low back pain: longitudinal data from the Norwegian HUNT Study
}

\author{
Anne Lovise Nordstoga, ${ }^{1}$ Tom Ivar Lund Nilsen, ${ }^{1}$ Ottar Vasseljen, ${ }^{1}$ \\ Monica Unsgaard-Tøndel, ${ }^{1,2}$ Paul Jarle Mork ${ }^{1}$
}

To cite: Nordstoga AL, Nilsen TIL, Vasseljen 0, et al. The influence of multisite pain and psychological comorbidity on prognosis of chronic low back pain: Iongitudinal data from the Norwegian HUNT Study. BMJ Open 2017;0:e015312. doi:10.1136/ bmjopen-2016-015312

- Prepublication history for this paper is available online. To view these files please visit the journal online (http://dx.doi org/10.1136/bmjopen-2016015312).

Received 29 November 2016 Revised 21 March 2017 Accepted 24 April 2017

\section{CrossMark}

${ }^{1}$ Department of Public Health and Nursing, Faculty of Medicine and Health Sciences, Norwegian University of Science and Technology, Trondheim, Norway ${ }^{2}$ Department of Neuromedicine and Movement Science,

Faculty of Medicine and Health Sciences, Norwegian University of Science and Technology, Trondheim, Norway

Correspondence to Dr Anne Lovise Nordstoga; anne.I.nordstoga@ntnu.no

\section{ABSTRACT}

Objectives This study aimed to investigate the prospective influence of multisite pain, depression, anxiety, self-rated health and pain-related disability on recovery from chronic low back pain (LBP).

Setting The data is derived from the second (1995-1997) and third (2006-2008) wave of the Nord-Trøndelag Health Study (HUNT) in Norway.

Participants The study population comprises 4484 women and 3039 men in the Norwegian HUNT Study who reported chronic LBP at baseline in 1995-1997.

Primary outcome measures The primary outcome was recovery from chronic LBP at the 11-year follow-up. Persons not reporting pain and/or stiffness for at least three consecutive months during the last year were defined as recovered. A Poisson regression model was used to estimate adjusted risk ratios (RRs) with 95\% Cls. Results At follow-up, 1822 (40.6\%) women and 1578 $(51.9 \%)$ men reported recovery from chronic LBP. The probability of recovery was inversely associated with number of pain sites ( $P$-trend $<0.001)$. Compared with reporting 2-3 pain sites, persons with only LBP had a slightly higher probability of recovery (RR $1.10,95 \% \mathrm{Cl}$ 0.98 to 1.22 in women and $\mathrm{RR} 1.10,95 \% \mathrm{Cl} 1.01$ to 1.21 in men), whereas people reporting 6-9 pain sites had substantially lower probability of recovery (RR $0.58,95 \% \mathrm{Cl}$ 0.52 to 0.63 in women and $\mathrm{RR} 0.70,95 \% \mathrm{Cl} 0.63$ to 0.79 in men). Poor/not so good self-rated general health, symptoms of anxiety and depression, and pain-related disability in work and leisure were all associated with reduced probability of recovery, but there was no statistical interaction between multisite pain and these comorbidities. Conclusions Increasing number of pain sites was inversely associated with recovery from chronic LBP. In addition, factors such as poor self-rated health, psychological symptoms and pain-related disability may further reduce the probability of recovery from chronic LBP.

\section{INTRODUCTION}

Low back pain (LBP) is a common cause of disability and is ranked as the most burdensome disease globally. ${ }^{12}$ LBP is the fourth most common diagnosis (after upper respiratory infection, hypertension and coughing) seen in primary care ${ }^{3}$ and approximately

\section{Strengths and limitations of this study}

- The strengths of the current study are the large and unselected population of women and men with chronic low back pain (LBP), the prospective design and the possibility of adjusting for several potential confounding factors.

- A limitation is the lack of information about the course of LBP and the other variables between the Nord-Trøndelag Health 2 (HUNT2) and HUNT3 Study.

- Furthermore, we cannot rule out that changes in lifestyle differed between those who experienced remission of symptoms and those who did not, for example, individuals with a high number of pain sites at baseline could be less prone to adopt a healthy lifestyle during the follow-up period because of the pain-related disability.

every fifth adult suffers from chronic LBP. ${ }^{4}$ Thus, in addition to the suffering experienced by affected individuals, LBP represents a substantial economic burden to the society. This underscore the importance of increased knowledge about factors that can improve the prevention and management of chronic LBP.

Chronic LBP rarely exists as a separate entity and co-occurrence of multisite pain and other comorbidities are common. ${ }^{5-9} \mathrm{~A}$ large case-control study comprising more than 100000 people showed that individuals with chronic LBP had higher occurrence of other musculoskeletal conditions, depression, anxiety and sleep disorders compared with controls without chronic LBP. $^{10}$ In particular, other chronic pain conditions are very prevalent among people with chronic LBP. ${ }^{5}$ Number of pain sites by itself has been suggested to bedose-dependently related to reduced physical and mental function ${ }^{11} 12$ and there are data to support the notion that generalised pain differs markedly from conditions with only one or a few pain sites with 
respect to other risk factors. ${ }^{13}$ Currently, there is a lack of longitudinal studies addressing how the extent of multisite pain influences the prognosis of chronic LBP. Moreover, it is unclear to what extent multisite pain interacts with other comorbid factors such as poor self-rated general health, pain-related disability and poor mental health to influence the prognosis of chronic LBP.

The main objective of this study was therefore to prospectively investigate the influence of common somatic and psychological comorbidities on prognosis of chronic LBP. We hypothesised (1) that multisite chronic pain, poor self-rated general health, pain-related disability and poor psychological health are factors that are inversely and independently related to the probability of recovery from chronic LBP and (2) that the possible association between number of pain sites and prognosis of LBP is modified by other somatic and psychological comorbidities.

\section{METHODS}

\section{Study population}

In Nord-Trøndelag county, Norway, all inhabitants aged 20 years or older were invited to participate in three health surveys (the Nord-Trøndelag Health Study (the HUNT Study)), the first in 1984-1986 (HUNT1), the second in 1995-1997 (HUNT2) and the last in 20062008 (HUNT3). The current study is based on data from HUNT2 and HUNT3. Of 93898 eligible participants, $65237(65.5 \%)$ accepted the invitation to participate in HUNT2. In HUNT3, a total of 93860 participants were invited, and 50807 (54.1\%) accepted the invitation. More detailed information about selection procedures, participation and questionnaires used in the HUNT Study can be found at http://www.ntnu.edu/hunt.

Information on lifestyle and health-related factors were collected by questionnaires and a clinical examination at both HUNT2 and HUNT3. For the purpose of this study, we included data from the 37070 people who participated at both surveys. We excluded 15062 women and 12861 men who reported to be free from chronic LBP at HUNT2. Moreover, we excluded 1557 persons with missing information on musculoskeletal pain at HUNT3 and 23 persons without complete values on body mass index (BMI) from the clinical examination. Further, 44 persons defined as underweight (BMI $<18.5 \mathrm{~kg} / \mathrm{m}^{2}$ ) were additionally excluded from the analyses to reduce the possibility for reverse causation due to undetected disease. Thus, the prospective analyses were based on 4484 women and 3039 men. Each participant signed a written consent, and the study was approved by the Regional Committee for Ethics in Medical Research (project no. 2014/2044 REK midt, Norway).

\section{Chronic LBP}

The questions about musculoskeletal pain were adopted from the Standardised Nordic Questionnaire. ${ }^{14}$ The participants were asked "During the last year, have you had pain and/or stiffness in your muscles and joints that lasted for at least three consecutive months?" Response options were 'yes' and 'no'. If answering 'yes', the participants were asked to indicate the affected body area(s), that is, up to nine body areas (neck, shoulders/upper arms, upper back, elbows, low back, wrists/hands, hips, knees and ankles/feet). Chronic LBP was in both surveys defined by 'yes' to the first question and low back indicated as an affected body area by the second question. Persons who responded 'yes' to the first question but did not indicate low back as an affected body area were considered to be free from chronic LBP. Number of chronic pain sites were estimated by adding together pain-afflicted body areas, of which the total number of pain sites includes LBP. The primary outcome was recovery from chronic LBP at the 11-year follow-up. Persons categorised with chronic LBP at HUNT2 responding 'no' at HUNT3 to the question "During the last year, have you had pain and/or stiffness in your muscles and joints that lasted for at least three consecutive months?" were defined as recovered.

\section{Pain-related comorbidities}

The participants' self-rated general health was evaluated using the question "How is your health at the moment?", with response options 'very good', 'good', 'not so good' and 'poor'. The answers were dichotomised into two groups: 'very good/good' and 'not so good/poor' in line with previous studies. ${ }^{15}$

Pain-related disability was evaluated separately for work ability and leisure time activity. The question about work ability was: "Have the pain and/or stiffness reduced your ability to work during the last year?" with four possible responses: 'no', 'not significantly', 'to some degree', 'significantly' and 'don't know'. The first and last response options were merged and categorised as 'no disability', and the two middle categories as 'work disability'. For leisure time activity, the question was: "Have the pain/ or stiffness reduced your leisure activity?" with possible responses: 'yes' and 'no'. The responses on disability due to musculoskeletal symptoms were then categorised into four groups: 'no disability', 'work disability', 'leisure disability' and 'work and leisure disability'.

Symptoms of anxiety and depression were measured using the Hospital Anxiety and Depression Scale (HADS). HADS is a validated and well-established self-rating questionnaire including seven questions on anxiety and seven questions on depression. ${ }^{16}$ As recommended, the cut-off score value was set to $\geq 8$ on both anxiety and depression and were dichotomised as presence or no presence of anxiety and/or depression..$^{1617}$ In addition, a mixed HADS variable was constructed consisting of four groups: 'no depression or anxiety', 'only depression', 'only anxiety' and 'both depression and anxiety'. ${ }^{18}$ Symptoms of only depression or only anxiety were defined by a HADS score $\geq 8$ on the respective subscales, whereas symptoms of both depression and anxiety were defined by a HADS score $\geq 8$ on both subscales. 


\section{Possible confounders}

All estimated associations were adjusted for possible confounders. Age was categorised in 20-29, $30-39, \ldots \geq 60$ years. BMI was calculated as weight divided by the square of height $\left(\mathrm{kg} / \mathrm{m}^{2}\right)$ by standardised measurements of height and weight from the clinical examination, and classified into BMI groups according to the suggestions by the WHO (normal weight, overweight and obesity). ${ }^{19}$ Physical work demands were assessed by the question: "If you have paid or unpaid work, how would you describe your work?" with the possible responses: 'mostly sedentary', 'much walking', 'much walking and lifting' or 'heavy physical work'. Leisure time physical activity was assessed by the question: "How much of your leisure time have you been physically active during the last year?" where the participants reported number of hours of light and/or hard activity. Four categories were constructed based on this information; 'inactive' (no light or hard activity), 'low activity' ( $<3$ hours of light and no hard activity), 'moderate activity' ( $\geq 3$ hours light and/or $<1$ hours of hard activity) and 'high activity' (any light and $\geq 1$ hour of hard activity). Further, education was assessed by the question "what is your highest level of education?", and were divided in four categories: 'primary school', 'high school', 'college $\leq 4$ years' and 'college $>4$ years'. Smoking was assessed by questions about past or present use of cigarettes, and were divided in three categories: 'never-smoker', ' previous smoker' and 'current smoker'.

\section{Statistical analysis}

We used a generalised linear model of the Poisson family to estimate the relative probability of recovery from chronic LBP as risk ratios (RRs) with 95\% CIs. An RR $>1.0$ indicates higher probability of recovery compared with the reference category, whereas a $\mathrm{RR}<1.0$ indicates a reduced probability of recovery. All estimated associations were adjusted for age, BMI, physical activity, education, smoking and physical work demands. All main analyses were conducted separately for men and women. Furthermore, a test for linear trend (ie, dose response) across categories of number of pain sites was conducted by treating the categories as an ordinal variable in the regression model.

In addition, we conducted analyses combining number of pain sites (<4vs 4-9 sites) and comorbid conditions in relation to the probability of recovery from chronic LBP. Previous studies have shown that reporting of $\geq 4$ pain sitesis associated with a markedly poorer prognosis of pain relief, ${ }^{20}$ as well as increasing likelihood of healthcare utilisation and sickness absence. ${ }^{21}$ Statistical interaction was evaluated by likelihood ratio tests of a product term of number of pain sites and each of the comorbid factors (self-reported health, pain-related disability and HADS). All statistical analyses were performed using Stata for Windows V.13.1.

\section{RESULTS}

Table 1 presents the baseline characteristics of the study population according to number of chronic pain sites. At baseline, $66.4 \%$ of the women and $47.2 \%$ of the men reported $\geq 4$ pain sites. Of the 4484 women and 3039 men who reported chronic LBP at baseline (HUNT2), 1822 (40.6\%) women and 1578 (51.9\%) men were reported recovered from chronic LBP at the 11-year follow-up (HUNT3).

Table 2 shows the association between number of pain sites, pain-related disability, psychological symptoms and self-rated general health with the probability of recovery from chronic LBP at follow-up. Increasing number of pain sites was inversely associated with the probability of recovery (P-trend $<0.001$ in both women and men). In specific, women and men who reported 6-9 pain sites had substantially lower probability of recovery (RR $0.58,95 \%$ CI 0.52 to 0.63 and RR $0.70,95 \%$ CI 0.63 to 0.79 , respectively), compared with women and men who reported 2-3 pain sites. People with only LBP had a

Table 1 Baseline characteristics of the study population stratified by gender and number of chronic pain sites

\begin{tabular}{|c|c|c|c|c|}
\hline & \multicolumn{2}{|l|}{ Women } & \multicolumn{2}{|l|}{ Men } \\
\hline & $<4$ pain sites & 4-9 pain sites & $<4$ pain sites & 4-9 pain sites \\
\hline No of persons (\%) & $1506(33.6)$ & $2978(66.4)$ & 1605 (52.8) & $1434(47.2)$ \\
\hline Body mass index $\left(\mathrm{kg} / \mathrm{m}^{2}\right)$, mean (SD) & $26.1(4.1)$ & $27.0(4.5)$ & $26.5(3.3)$ & $27.0(3.4)$ \\
\hline Physically inactive, n (\%) & $82(5.4)$ & $208(7.0)$ & $96(6.0)$ & $103(7.2)$ \\
\hline Current smoker, n (\%) & $427(28.4)$ & $1021(34.3)$ & $416(25.9)$ & $412(28.7)$ \\
\hline Poor/not so good self-rated health, $n(\%)$ & $443(29.4)$ & $1786(60.0)$ & $461(28.7)$ & $831(57.9)$ \\
\hline Pain-related disability, work and leisure, n (\%) & 726 (48.2) & $2034(68.3)$ & $784(48.8)$ & $970(67.6)$ \\
\hline HADS score depression >8, n (\%) & $65(4.3)$ & $187(6.3)$ & $96(6.0)$ & $124(8.6)$ \\
\hline HADS score anxiety >8, n (\%) & $149(9.9)$ & $425(14.3)$ & $110(6.9)$ & $147(10.3)$ \\
\hline
\end{tabular}

HADS, Hospital Anxiety and Depression Scale. 


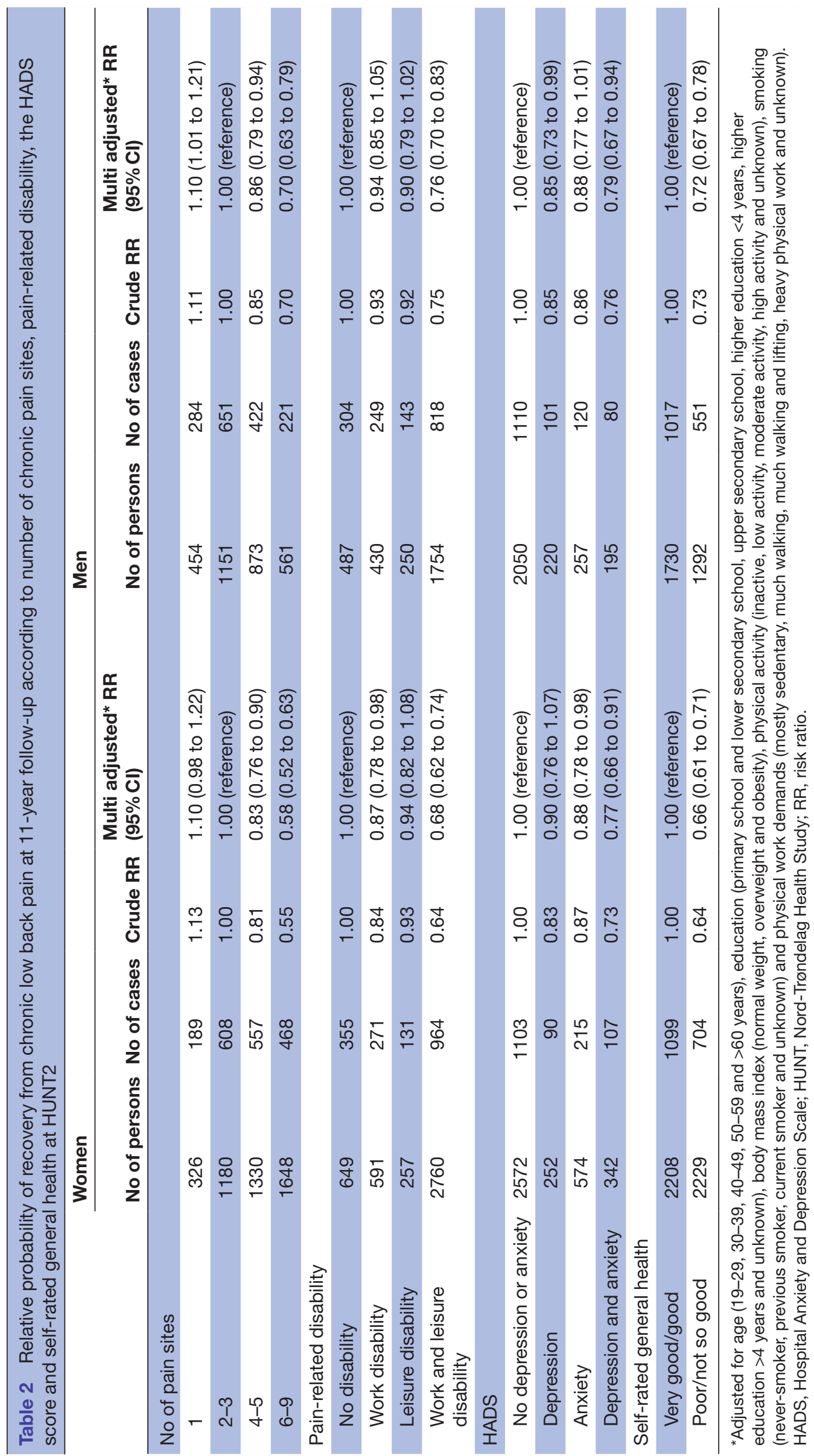


slightly higher probability of recovery (RR 1.10, 95\% CI 0.98 to 1.22 in women and RR $1.10,95 \%$ CI 1.01 to 1.21 in men) compared with women and men who reported 2-3 pain sites. Pain-related disability that influenced both work ability and leisure activity was associated with reduced probability of recovery in both women (RR $0.68,95 \%$ CI 0.62 to 0.74 ) ) and men (RR $0.76,95 \%$ CI 0.70 to 0.83 ). HADS score $\geq 8$ on both depression and anxiety subscales was associated with reduced probability of recovery in both women (RR $0.77,95 \%$ CI 0.66 to 0.91 ) and men (RR $0.79,95 \%$ CI 0.67 to 0.94 ). Persons reporting poor or not so good general health had a markedly reduced probability of recovery, both in women (RR $0.66,95 \%$ CI 0.61 to 0.71 ) and men (RR $0.72,95 \%$ CI 0.67 to 0.78 ), compared with those reporting good or very good general health.

Table 3 presents the combined effect of number of pain sites and pain-related disability, psychological symptoms and self-rated general health on the probability of recovering for chronic LBP. We did not observe any statistical interaction between number of pain sites and pain-related disability, psychological symptoms or self-rated health ( $\mathrm{p} \geq 0.24$ for all tests). However, stratified analysis within categories of the exposure variables showed that reporting $\geq 4$ pain sites was associated with lower probability of recovery independently of level of pain-related disability and psychological symptoms. Within strata of pain-related disability, persons who reported $\geq 4$ pain sites had 16\%-27\% lower probability of remission compared with persons with 1-3 pain sites in the same pain-related disability categories. Likewise, within the different strata of psychological symptoms, persons with $\geq 4$ pain sites had $25 \%-35 \%$ lower probability of recovery compared with persons with 1-3 pain sites.

\section{DISCUSSION}

In this large population-based study, we found that musculoskeletal comorbidity, reduced self-rated general health and psychological symptoms were independently associated with reduced probability of recovery from chronic LBP at 11-year follow-up. The factors with the strongest association with poor prognosis were widespread chronic pain (6-9 pain sites) and poor or not so good self-rated general health. The strength of the associations between the various comorbidities and pain prognosis was fairly similar for women and men. Probability of recovery from chronic LBP was inversely associated with increasing number of chronic pain sites. Although there was no interaction between number of chronic pain sites and other comorbidities, we observed in the combined analysis that persons with $\geq 4$ pain sites were associated with lower probability of recovery from chronic LBP within all strata of pain-related disability and symptoms of depression and/or anxiety. The current findings indicate that musculoskeletal comorbidity has a strong and independent influence on long-term prognosis of chronic LBP.
It is noteworthy that about $66 \%$ of the women and $47 \%$ of the men in this study reported $\geq 4$ chronic pain sites at baseline. This supports the view that co-occurrence of musculoskeletal pain is very common in chronic LBP. ${ }^{56}$ To our knowledge, this is the first population-based study to investigate the prospective influence of graded musculoskeletal comorbidity on the prognosis of chronic LBP. The dose-response association between number of chronic pain sites and reduced probability of recovery from chronic LBP suggests that musculoskeletal comorbidity should be considered an important predictor in identifying target groups for public health secondary prevention. This was also supported by our combined analysis, showing that number of pain sites was the main driving factor for predicting persistence of chronic LBP.

More than $40 \%$ of the women and $50 \%$ of the men in the current study reported recovery from chronic LBP at 11-year follow-up. Interestingly, a previous study showed that the prevalence of chronic LBP was relatively stable from HUNT2 to HUNT3 with about $26 \%$ of women and $20 \%$ of men reporting chronic LBP at both surveys. ${ }^{22}$ Thus, our results indicate that during an 11-year period a substantial proportion of the population shift from having chronic LBP to remission, but that a substantial proportion also develops pain in the same period. Similar large fluctuations in reporting of chronic LBP at the individual level have also been observed by others. ${ }^{23}{ }^{24}$ Thus, our findings lend further support to the notion that chronic LBP on the individual level may fluctuate substantially over time while the population prevalence remains relatively stable. The current study adds to this knowledge by showing that individuals who shift from having chronic LBP symptoms to remission of symptoms are more likely to have fewer chronic pain sites, less pain-related disability, better self-rated health and no major symptoms of anxiety or depression.

Increasing number of chronic pain sites were inversely associated with probability of recovery, that is, women and men who reported $\geq 6$ pain sites had about $30 \%-40 \%$ lower probability of recovery from chronic LBP compared with women and men with 2-3 pain sites. Previous cross-sectional studies have indicated a dose-response association between number of pain sites and a range of negative health outcomes such as psychological distress, poor sleep, poor self-rated health, reduced social and functional ability, ${ }^{11}$ as well as increased sickness absence and healthcare utilisation. ${ }^{25}$ The current prospective study extends this body of knowledge showing that number of chronic pain sites have a strong dose-response influence on prognosis of chronic LBP. Although we observed no interaction between number of chronic pain sites and other comorbid factors, the probability of relief from chronic LBP was consistently lower for the group with multisite pain within all strata of pain-related disability and psychological symptoms scores. These findings support the long-held view that it may be useful to classify patients with chronic LBP into 'back pain alone' or 'back pain plus other pain' to improve clinical decision-making. ${ }^{26}$ 


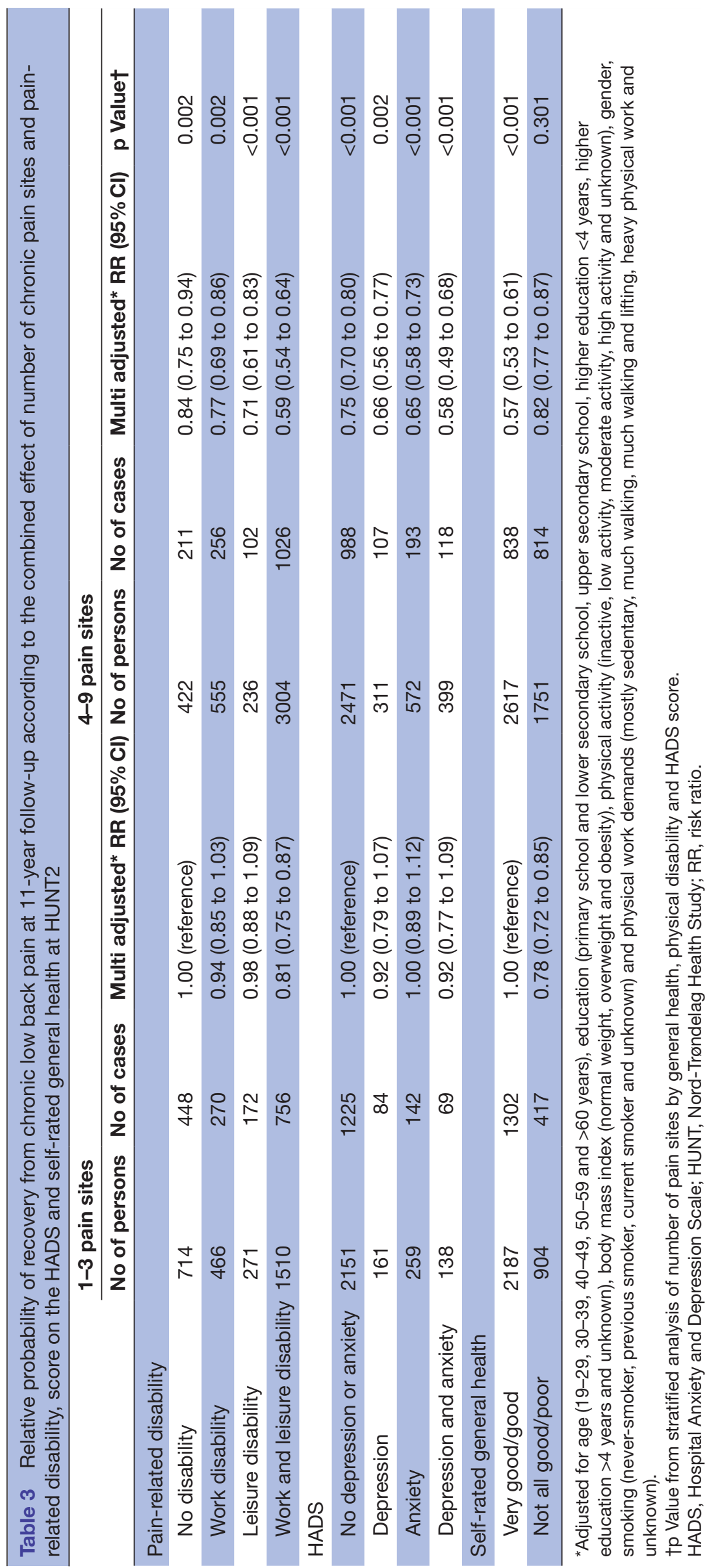


The current finding of a dose-response association between number of chronic pain sites and prognosis of chronic LBP may indicate that the extent of musculoskeletal comorbidity could provide additional complementary information to improve classification in stratified care approaches. The idea that assessment of multisite pain can assist clinical judgement of prognosis and improve targeted treatment has been proposed before, ${ }^{6}$ and the current data lend further support to this idea. Furthermore, since number of chronic pain sites per se seem to be a strong prognostic factor in chronic LBP it may also be useful to consider this variable when recruiting subjects into research studies to facilitate baseline comparisons.

Although previous data indicate that psychological symptoms are more common in patients with LBP than in comparable controls, ${ }^{10}$ our results do not indicate that such symptoms strongly influence the prognosis of chronic LBP. However, another study of subjects with neck and/or LBP in HUNT3 showed that symptoms of mental distress were significant determinants for seeking healthcare, which could have moderated the associations. ${ }^{27}$ Our findings are in line with Dunn $e t a l^{28}$ who found no significant association between depression, and only a modest association between anxiety, and the risk of disabling LBP at 12 months follow-up in patients presenting with LBP in general practice. In the same study, it was observed that self-rated health had a relatively strong impact on prognosis of LBP with patients who rated their health as poor having more than twofold increased risk of disabling back pain. Very few individuals in our study population rated their health as poor and we were, therefore, not able to estimate probability for recovery among these individuals. However, we observed that women and men who rated their health as less than good (ie, poor or not so good) had about $30 \%$ lower probability of recovery from chronic LBP compared with those who rated their health as good or very good.

The strengths of the current study are the large and unselected population of women and men with chronic LBP, the prospective design and the possibility of adjusting for several potential confounding factors. The questions on chronic musculoskeletal pain used in HUNT2 have acceptable reliability and validity. ${ }^{14} 2930$ Likewise, the HADS Scale has been shown to be at a valid indicator of possible depression and anxiety in clinical practice as well as in the general population. ${ }^{16} 1731$ A limitation is the lack of follow-up information about the course of LBP and the other variables between the HUNT2 and HUNT3 Study. Thus, any changes occurring during the follow-up period could not be taken into account in the analyses. For example, information regarding treatment during the follow-up period or information on changes in lifestyle could be of interest. A healthy lifestyle has been associated with improved long-term outcome in individuals with recurrent LBP episodes. ${ }^{32}$ Thus, it may be possible that individuals who changed their lifestyle during the follow-up period also altered their course of chronic LBP. Furthermore, we cannot rule out that such changes in lifestyle were differential between participants who experienced remission of symptoms versus those who did not, for example, individuals with a high number of pain sites at baseline could be less prone to adopt a healthy lifestyle during the follow-up period because of pain-related disability.

In conclusion, the current study indicates that multisite chronic pain is independently and inversely associated with the probability of recovery from chronic LBP. Poor self-rated health, psychological symptoms and pain-related disability might further reduce the probability of recovery from chronic LBP. There was no interaction between number of chronic pain sites and other comorbidities, including pain-related disability, psychological symptoms and self-rated general health. These findings underscore the importance of taking comorbid symptoms into account, and in particular number of chronic pain sites, when designing management programmes or treatment for secondary prevention of chronic LBP.

Acknowledgements The HUNT Study is a collaboration between the HUNT Research Centre (Faculty of Medicine, Norwegian University of Science and Technology), Nord-Trøndelag County Council and the Norwegian Institute of Public Health.

Contributors PJM, TILN and ALN designed the study, and all authors contributed in the discussion of the design and interpretation. ALN performed the analyses supervised by PJM and TILN. The first draft of the manuscript was written by PJM and ALN. All authors contributed to the final manuscript.

Funding This study was supported by The Norwegian Fund for Postgraduate Training in Physiotherapy and by grants from the Liaison Committee between the Central Norway Regional Health Authority and the Norwegian University of Science and Technology (NTNU).

Competing interests None declared.

Patient consent Obtained.

Ethics approval The current study was approved by the Regional Committee for Ethics in Medical Research (project no. 2014/2044 REK midt, Norway). The study was carried out according to the Declaration of Helsinki.

Provenance and peer review Not commissioned; externally peer reviewed. Data sharing statement No additional data available.

Open Access This is an Open Access article distributed in accordance with the Creative Commons Attribution Non Commercial (CC BY-NC 4.0) license, which permits others to distribute, remix, adapt, build upon this work non-commercially, and license their derivative works on different terms, provided the original work is properly cited and the use is non-commercial. See: http://creativecommons.org/ licenses/by-nc/4.0/

(c) Article author(s) (or their employer(s) unless otherwise stated in the text of the article) 2017. All rights reserved. No commercial use is permitted unless otherwise expressly granted.

\section{REFERENCES}

1. Murray CJ, Vos T, Lozano R, et al. Disability-adjusted life years (DALYs) for 291 diseases and injuries in 21 regions, 1990-2010: a systematic analysis for the global burden of disease study 2010. Lancet 2012;380:2197-223.

2. Vos T, Flaxman AD, Naghavi M, et al. Years lived with disability (YLDs) for 1160 sequelae of 289 diseases and injuries 1990-2010: a systematic analysis for the global burden of disease study 2010 . Lancet 2012;380:2163-96.

3. Wändell P, Carlsson AC, Wettermark B, et al. Most common diseases diagnosed in primary care in Stockholm, Sweden, in 2011. Fam Pract 2013;30:506-13.

4. Hoy D, Bain C, Williams G, et al. A systematic review of the global prevalence of low back pain. Arthritis Rheum 2012;64:2028-37. 
5. Von Korff M, Crane P, Lane M, et al. Chronic spinal pain and physical-mental comorbidity in the United States: results from the national comorbidity survey replication. Pain 2005;113:331-9.

6. Hartvigsen J, Natvig B, Ferreira M. Is it all about a pain in the back? Best Pract Res Clin Rheumatol 2013;27:613-23.

7. Croft P. The question is not "have you got it"? But "how much of it have you got"? Pain 2009;141:6-7.

8. Hestbaek L, Leboeuf-Yde C, Manniche C. Is low back pain part of a general health pattern or is it a separate and distinctive entity? A critical literature review of comorbidity with low back pain. $J$ Manipulative Physiol Ther 2003;26:243-52.

9. Ramond-Roquin A, Pecquenard F, Schers $\mathrm{H}$, et al. Psychosocial, musculoskeletal and somatoform comorbidity in patients with chronic low back pain: original results from the dutch transition project. Fam Pract 2015;32:297-304.

10. Gore M, Sadosky A, Stacey BR, et al. The burden of chronic low back pain: clinical comorbidities, treatment patterns, and health care costs in usual care settings. Spine 2012;37:E668-77.

11. Kamaleri $Y$, Natvig B, Ihlebaek CM, et al. Number of pain sites is associated with demographic, lifestyle, and health-related factors in the general population. Eur J Pain 2008;12:742-8.

12. Kamaleri $Y$, Natvig B, Ihlebaek CM, et al. Localized or widespread musculoskeletal pain: does it matter? Pain 2008:138:41-6.

13. Coggon D, Ntani G, Palmer KT, et al. Patterns of multisite pain and associations with risk factors. Pain 2013;154:1769-77.

14. Kuorinka I, Jonsson B, Kilbom A, et al. Standardised Nordic Questionnaires for the analysis of musculoskeletal symptoms. Appl Ergon 1987;18:233-7.

15. Krokstad S, Kunst AE, Westin S. Trends in health inequalities by educational level in a Norwegian total population study. J Epidemiol Community Health 2002;56:375-80.

16. Bjelland I, Dahl AA, Haug TT, et al. The validity of the Hospital Anxiety and Depression Scale. An updated literature review. J Psychosom Res 2002;52:69-77.

17. Lisspers J, Nygren A, Söderman E, et al. Hospital Anxiety and Depression Scale (HAD): some psychometric data for a Swedish sample. Acta Psychiatr Scand 1997;96:281-6.

18. Mundal I, Gråwe RW, Bjørngaard JH, et al. Prevalence and longterm predictors of persistent chronic widespread pain in the general population in an 11-year prospective study: the HUNT study. BMC Musculoskelet Disord 2014;15:213.

19. Physical status: the use and interpretation of anthropometry. Report of a WHO Expert Committee. World Health Organ Tech Rep Ser $1995 ; 854: 1-452$
20. Vasseljen O, Woodhouse A, Bjørngaard $\mathrm{JH}$, et al. Natural course of acute neck and low back pain in the general population: the HUNT study. Pain 2013;154:1237-44.

21. de Fernandes RC, Burdorf A. Associations of multisite pain with healthcare utilization, sickness absence and restrictions at work. Int Arch Occup Environ Health 2016;89:1039-46.

22. Hagen $\mathrm{K}$, Linde M, Heuch I, et al. Increasing prevalence of chronic musculoskeletal complaints. A large 11-year follow-up in the general population (HUNT 2 and 3). Pain Med 2011;12:1657-66.

23. Hestbaek L, Leboeuf-Yde C, Engberg M, et al. The course of low back pain in a general population. results from a 5-year prospective study. J Manipulative Physiol Ther 2003;26:213-9.

24. Tamcan O, Mannion AF, Eisenring C, et al. The course of chronic and recurrent low back pain in the general population. Pain 2010;150:451-7.

25. de Fernandes RC, Burdorf A. Associations of multisite pain with healthcare utilization, sickness absence and restrictions at work. Int Arch Occup Environ Health 2016;89:1039-46. Epub ahead of print.

26. Natvig B, Bruusgaard D, Eriksen W. Localized low back pain and low back pain as part of widespread musculoskeletal pain: two different disorders? A cross-sectional population study. J Rehabil Med 2001;33:21-5.

27. Woodhouse A, Pape K, Romundstad PR, et al. Health care contact following a new incident neck or low back pain episode in the general population; the HUNT study. BMC Health Serv Res 2016;16:81.

28. Dunn KM, Jordan KP, Croft PR. Contributions of prognostic factors for poor outcome in primary care low back pain patients. Eur J Pain 2011;15:313-9.

29. Descatha A, Roquelaure Y, Chastang JF, et al. Validity of Nordicstyle questionnaires in the surveillance of upper-limb workrelated musculoskeletal disorders. Scand $J$ Work Environ Health 2007;33:58-65.

30. Palmer K, Smith G, Kellingray S, et al. Repeatability and validity of an Upper Limb and Neck Discomfort Questionnaire: the utility of the standardized Nordic Questionnaire. Occup Med 1999;49:171-5.

31. Østby-Deglum I, Mykletun A, Dahl AA. The Hospital anxiety and depression Rating Scale (HADS) as a case finder for anxiety disorder and depression in norwegian general practices. Acta Psychiatrica Scandinavica 2004;110:43-4.

32. Bohman T, Alfredsson L, Jensen I, et al. Does a healthy lifestyle behaviour influence the prognosis of low back pain among men and women in a general population? A population-based cohort study. BMJ Open 2014;4:e005713. 